\title{
História, resolução numérica e GeoGebra no ensino de equações algébricas
}

\author{
Rubens Ortega Thiago Abbeg
}

\begin{abstract}
Resumo
Este trabalho propõe uma metodologia alternativa para o estudo tradicional de Equações Algébricas, sugerindo a consideração de três componentes: visão histórica do tema, implementação de um método numérico, o da Bisseção, e utilização do aplicativo GeoGebra. Tem como objetivo principal completar uma lacuna presente na formação dos estudantes do Ensino Médio, pois, basicamente, os métodos de resolução ali apresentados permitem apenas encontrar raízes racionais das equações. Raízes irracionais, de igual importância, são praticamente deixadas de lado. A ideia foi desenvolvida em uma Dissertação de Mestrado do Profmat ${ }^{1}$ [1], e segue o pensamento exposto em $[4]$.
\end{abstract}

Palavras-chave: Ensino de Matemática; Equações Algébricas; Método da Bisseção; GeoGebra.

\section{Abstract}

This work proposes an alternative methodology for the traditional study of Algebraic Equations, suggesting the consideration of three components: historical view of the theme, implementation of a numerical method, the Bisection, and use of the GeoGebra application. Its main objective is to fill a gap in the training of high school students, because basically the methods of resolution presented there allow only to find rational roots of the equations. Irrational roots, of same importance, are practically neglected. The idea was developed in a Master's Dissertation of the Profmat [1] and follows the thought exposed in [4].

Keywords: Mathematics Teaching; Algebraic Equations; Bisection method; GeoGebra.

\section{Introdução}

Não faz muito tempo, um mestre de obras consultou-nos sobre um financiamento que pretendia fazer na compra de um carro. Sua dúvida era se a prestação de $\mathrm{R} \$ 852,43$, anunciada pelo vendedor da concessionária, estava correta, já que o valor a ser financiado em 36 meses seria $\mathrm{R} \$ 25.000,00$, com taxa de juro de 0, 99\% ao mês. Com ajuda de uma calculadora, dissemos-lhe que, naquelas condições, a prestação deveria ser $\mathrm{R} \$ 828,93$. Ele pensou um pouco e, em seguida, perguntou qual

\footnotetext{
${ }^{1}$ Mestrado Profissional em Matemática em Rede Nacional - www.profmat-sbm.org.br/
} 
era, então, a taxa de juro que estava sendo cobrada para a prestação informada pelo vendedor. Foilhe dito que esta resposta não poderia ser dada naquele momento, e explicado que o problema de encontrar a taxa de juro é muito mais sofisticado do que o de encontrar a prestação. O resultado foi dado no dia seguinte, obtido com ajuda da função TAXA do $\mathrm{Excel}^{2}$, que forneceu o valor 1,1529\%, bem acima do anunciado $0,99 \%$.

Para responder a essas perguntas, consideremos a fórmula do Valor Atual [13],

$$
A=P \frac{1-(1+i)^{-n}}{i} \Leftrightarrow P=\frac{A i(1+i)^{n}}{(1+i)^{n}-1},
$$

onde $A$ é o valor a ser financiado, $P$ é a prestação, $i$ é a taxa de juro e $n$ é o número de parcelas. Conhecidos $A, i$ e $n$, pode-se facilmente encontrar $P$. No nosso caso, fica

$$
P=\frac{25000 \cdot 0,0099(1+0,0099)^{36}}{(1+0,0099)^{36}-1},
$$

que dá $P=\mathrm{R} \$ 828,93$. Por outro lado, dados $A, P$ e $n$, para encontrar o valor de $i$ somos levados ao problema de resolver uma equação algébrica de grau $n+1$, com termo independente nulo, isto é,

$$
A=P \frac{1-(1+i)^{-n}}{i} \Leftrightarrow(1+i)^{n} \cdot\left(\frac{A}{P} i-1\right)+1=0
$$

No nosso caso, fica

$$
(1+i)^{36} \cdot\left(\frac{25000}{852,43} i-1\right)+1=0 .
$$

O episódio anteriormente descrito fez-nos refletir sobre ser esse mais um importante assunto que o estudante não aprende nos 12 anos que passa pela Educação Básica. Durante a vida, a grande maioria das pessoas fará um financiamento, para comprar um carro, uma casa, um eletrodoméstico ou qualquer outro bem. Assim sendo, o cálculo da taxa de juro deveria fazer parte dos conhecimentos básicos de cada pessoa, inclusive para o bom exercício da cidadania. Dessa forma, como tal cálculo e muitos outros oriundos de problemas importantes [4] fazem parte do tema "Resolução de Equações Algébricas", acreditamos ser de grande relevância tratar do assunto em sala de aula fazendo uso de uma abordagem mais geral e moderna.

\section{Equações algébricas no ensino médio}

Os conteúdos relacionados às Equações Algébricas são tratados na $3^{a}$ série do Ensino Médio, logo após o estudo dos Números Complexos. Os principais resultados [8] apresentados aos estudantes são enunciados a seguir.

Teorema Fundamental da Álgebra: Todo polinômio $P$ de grau $n \geq 1$ admite ao menos uma raiz complexa, podendo ser real.

Teorema das Raízes Complexas: Se uma equação algébrica de coeficientes reais admite como raiz o número complexo $z=\alpha+\beta i(\beta \neq 0)$, então essa equação também admite como raiz o número $\bar{z}=\alpha-\beta i$.

\footnotetext{
${ }^{2}$ Editor de planilhas produzido pela Microsoft.
} 
Teorema de Bolzano: Sejam $P(x)=0$ uma equação algébrica com coeficientes reais e $(a, b)$ um intervalo real aberto. Se $P(a) \cdot P(b)>0$, então a equação possui um número par de raízes reais (ou não possui raiz) em $(a, b)$. Se $P(a) \cdot P(b)<0$, então a equação possui um número ímpar de raizes reais em $(a, b)$.

Teorema das Raízes Racionais: Se uma equação algébrica $a_{n} x^{n}+a_{n-1} x^{n-1}+a_{n-2} x^{n-2}+\cdots+$ $a_{1} x+a_{0}=0$, com $a_{n} \neq 0$, de coeficientes inteiros, admite uma raiz racional $p / q$, sendo $p$ e $q$ primos entre si, então $p$ é divisor de $a_{0}$ e q é divisor de $a_{n}$.

É com base nesse último resultado que os estudantes resolvem as equações que lhes são propostas. Por exemplo, para a equação $2 x^{3}+x^{2}-18 x-9=0$, as possíveis raízes inteiras são os divisores do termo independente $(-9)$, isto é, $\{-1,+1,-3,+3,-9,+9\}$, sendo que as possíveis raízes fracionárias são resultantes dos quocientes entre os elementos do conjunto anterior e os divisores do coeficiente do termo de mais alto grau (2), isto é, $\{-1,+1,-2,+2\}$. Com isso, conclui-se que o conjunto das possíveis raízes racionais da equação $2 x^{3}+x^{2}-18 x-9=0$ é $\{-1,+1,-3,+3,-9,+9,-1 / 2,+1 / 2,-3 / 2,+3 / 2,-9 / 2,+9 / 2\}$. A partir daí, a aplicação do dispositivo de Briot-Ruffini [8] permite encontrar, neste conjunto, basicamente por inspeção, quais são raízes. Neste caso, $\{-3,-1 / 2,+3\}$ é o conjunto solução. Por outro lado, o conjunto das possíveis raízes racionais da equação $P(x)=x^{3}+2 x-2=0$ é $\{-1,+1,-2,+2\}$, e, por simples verificação, constata-se que nenhuma delas é solução. Sabemos, também, pelo Teorema de Bolzano, que essa equação possui pelo menos uma raiz real no intervalo $(0,1)$, pois $P(0) \cdot P(1)<0$. Os estudantes concluem o Ensino Médio sem saber resolver, por exemplo, essa última equação.

Atualmente, existem aplicativos gratuitos, acessíveis ao público, que resolvem numericamente tais equações. Por exemplo, no versátil GeoGebra ${ }^{3}$, pode-se digitar a equação na Janela CAS e obter a solução. A Figura 1 mostra a solução da equação $2 x^{3}+x^{2}-18 x-9=0$, cujas raízes são todas racionais, e uma aproximação com dez casas decimais da única solução real da equação $P(x)=x^{3}+2 x-2=0$, que é irracional.

\footnotetext{
${ }^{3}$ Este aplicativo e diversos materiais relacionados podem ser obtidos em https://www.geogebra.org/. O presente trabalho não tem como objetivo detalhar as instruções para elaboração, no GeoGebra, das figuras que fazem parte do texto. Os leitores interessados na reprodução das atividades aqui descritas poderão acessá-las, inclusive para download, em https://www.geogebra.org/rubensortega.
} 


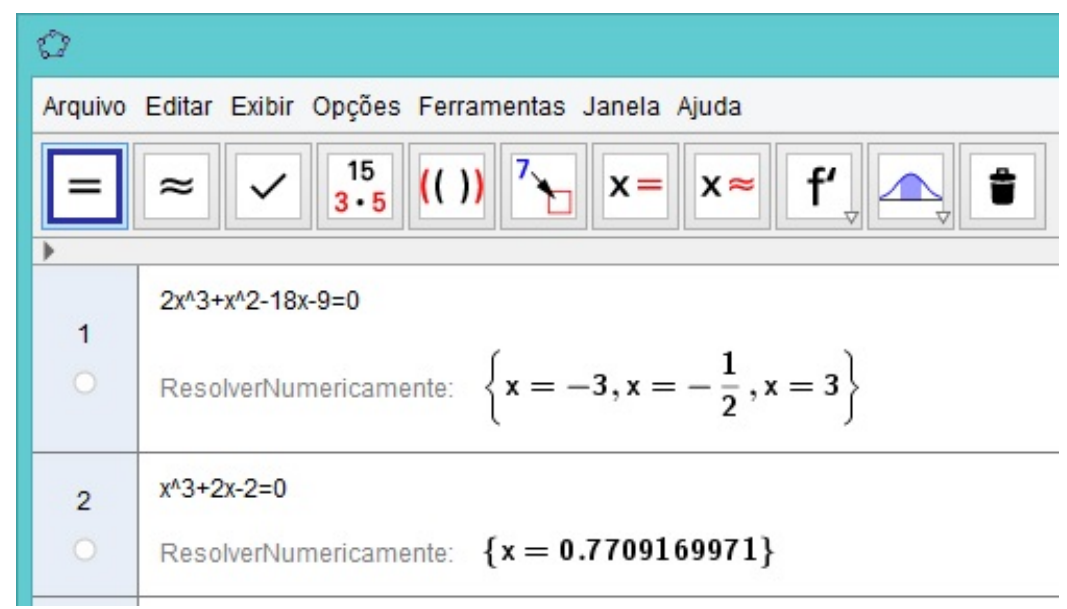

Figura 1: Resolução de Equações Algébricas no GeoGebra

Note-se que o programa funciona exatamente como uma calculadora e não mostra o caminho para se chegar ao resultado. Apesar de que conhecer essa ferramenta é de grande importância, vemos como muito mais interessante e pedagógico que o professor mostre aos estudantes um caminho matemático que faça com que se chegue à raiz, como foi no caso da equação $2 x^{3}+x^{2}-18 x-9=0$. Afinal, o próprio programa tem um algoritmo implementado que permite chegar à solução.

\section{Fórmula para a equação do $3^{\circ}$ grau}

O dia 10 de agosto de 1548 ficou marcado na História da Matemática pelo grande duelo matemático que travaram Niccolò Tartaglia (1499-1557) e Ludovido Ferrari (1522-1564), na igreja de Santa Maria do Jardim, em Milão, Itália. Esse duelo foi agendado após uma série de correspondências públicas trocadas entre os dois ilustres matemáticos, conhecida como Cartelli di Sfida Matematica ${ }^{4}$ [6], tudo em razão da famosa fórmula que resolve, por meio de radicais, a equação algébrica de terceiro grau

$$
a x^{3}+b x^{2}+c x+d=0,
$$

em função dos coeficientes $a, b, c$ e $d$.

A fórmula, que será apresentada nesta seção, foi publicada pela primeira vez na obra $[2]^{5}$, escrita em latim, de autoria de Gerolamo Cardano (1501-1576), tratado que figura entre os mais importantes da História da Matemática. Apesar de Cardano atribuir os créditos da descoberta da fórmula a Scipione Dal Ferro ${ }^{6}$ (1465-1526) e também a Tartaglia, este ficou enfurecido pois havia revelado o resultado a Cardano sob juramento de que o mesmo não o publicasse. A quebra da promessa, na visão de Tartaglia, foi imperdoável, e gerou um conflito de grande repercussão. No ano seguinte, Tartaglia publicou sua obra Quesiti et Inventione Diverse ${ }^{7}$ [17], na qual apresenta sua resolução

\footnotetext{
${ }^{4}$ Folhetos de Desafio Matemático.

${ }^{5}$ A Grande Arte, que possui edição traduzida para o inglês [3]

${ }^{6}$ Cardano encontrou manuscritos de Dal Ferro que comprovam que ele havia descoberto a fórmula antes de Tartaglia; porém, Dal Ferro nunca a publicou.

${ }^{7}$ Quesitos e Invenções Diversas, que possui edição traduzida para o francês [18]
} 
e faz duras críticas a Cardano. Em defesa de seu mestre, Ferrari rebateu publicamente as críticas de Tartaglia, o que gerou resposta de Tartaglia, réplica de Ferrari, e, após seis correspondências públicas (panfletos) escritas por cada um deles, finalmente o duelo matemático entre os dois foi marcado. Em razão de mais desavenças havidas entre os dois durante o embate, o duelo não prosseguiu até o final, ficando limitado apenas ao primeiro dia. Este desfecho foi frustrante e não houve um vencedor oficialmente declarado. Não obstante, o que ocorreu com a vida de cada um dos contendores após o episódio pode ser um indício de como a comunidade entendeu o resultado do confronto.

A solução por meio de radicais das equações do quarto grau foi demonstrada por Ferrari, e também publicada no Ars Magna. Posteriormente, Paolo Ruffini (1765-1822), Niels Henrik Abel (18021829) e Évariste Galois (1811-1832) foram os principais personagens envolvidos no estudo de equações de grau superior a quatro, demonstrando ser impossível resolvê-las por meio de radicais.

Após esta breve introdução histórica, passaremos ao procedimento que leva à fórmula que resolve as equações do $3^{\circ}$ grau, acreditando que, por sua simplicidade e valor histórico, deveria ser apresentada aos estudantes. Assim, queremos enfatizar que a inserção da História da Matemática como estratégia didática no processo de ensino-aprendizagem pode ser de grande valia. Particularmente, com relação às equações algébricas, praticamente tudo o que envolveu a descoberta das fórmulas e suas repercussões parece estar muito claro, pois existem referências que trazem dados precisos dos fatos, e que se baseiam em documentos históricos, como cartas e manuscritos redigidos pelos próprios protagonistas. Desta forma, como recurso didático a ser utilizado em suas aulas, recomendamos fortemente aos professores os excelentes livros $[7]^{8}$ e $[19]^{9}$, bem como os artigos [5], [9], [10] e [15].

Agora, seguindo a demonstração de [12], iremos encontrar uma fórmula que resolve a equação

$$
a x^{3}+b x^{2}+c x+d=0
$$

em função dos coeficientes $a, b, c$ e $d$.

Primeiro, notamos que se $A$ e $B$ são raízes da equação do segundo grau $z^{2}-S z+P=0$ logo $(A+B=S$ e $A B=P)$, então $y=\sqrt[3]{A}+\sqrt[3]{B}$ é solução da equação $y^{3}-3 \sqrt[3]{P} y-S=0$. De fato,

$$
\begin{gathered}
y^{3}=(\sqrt[3]{A}+\sqrt[3]{B})^{3}=A+B+3 \sqrt[3]{A B}(\sqrt[3]{A}+\sqrt[3]{B}) \\
y^{3}-3 \sqrt[3]{P} y-S=0
\end{gathered}
$$

Isto significa que, dada uma equação do terceiro grau do tipo $y^{3}+p y+q=0$, é possível escrever suas raízes como soma de raízes cúbicas das soluções de uma equação do segundo grau.

Para utilizar este resultado, primeiramente vamos transformar a equação que queremos resolver, isto é, $a x^{3}+b x^{2}+c x+d=0$, em outra sem o termo quadrático. Fazendo a mudança de variável $x=y+m$, obtemos

$$
\begin{array}{r}
a(y+m)^{3}+b(y+m)^{2}+c(y+m)+d=0 \\
a y^{3}+(3 a m+b) y^{2}+\left(3 a m^{2}+2 b m+c\right) y+\left(a m^{3}+b m^{2}+c m+d\right)=0 .
\end{array}
$$

\footnotetext{
${ }^{8}$ Este livro apresenta uma visão geral sobre a história das equações algébricas.

${ }^{9}$ Este livro aborda detalhes históricos em torno da fórmula que resolve as equações de terceiro grau.
} 
Se escolhermos $m=-\frac{b}{3 a}$, a equação reduz-se a

$$
a y^{3}+\left(3 a m^{2}+2 b m+c\right) y+\left(a m^{3}+b m^{2}+c m+d\right)=0,
$$

que é do tipo

$$
y^{3}+p y+q=0,
$$

$\operatorname{com} p=\frac{1}{a}\left(3 a m^{2}+2 b m+c\right)$ e $q=\frac{1}{a}\left(a m^{3}+b m^{2}+c m+d\right)$.

Comparando (1) com (2), obtemos $p=-3 \sqrt[3]{P}$ e $q=-S, \operatorname{logo} P=-\left(\frac{p}{3}\right)^{3}$ e $S=-q$.

A busca por dois números $\mathrm{A}$ e $\mathrm{B}$ cuja soma é $S=A+B$ e o produto é $P=A B$ leva-nos à resolução da equação do $2^{\circ}$ grau

$$
z^{2}-S z+P=0, \quad \text { ou } \quad z^{2}+q z-\left(\frac{p}{3}\right)^{3}=0
$$

Logo, $A=-\frac{q}{2}+\sqrt{\left(\frac{q}{2}\right)^{2}+\left(\frac{p}{3}\right)^{3}}$ e $B=-\frac{q}{2}-\sqrt{\left(\frac{q}{2}\right)^{2}+\left(\frac{p}{3}\right)^{3}}$. Finalmente,

$$
y=\sqrt[3]{A}+\sqrt[3]{B}=\sqrt[3]{-\frac{q}{2}+\sqrt{\left(\frac{q}{2}\right)^{2}+\left(\frac{p}{3}\right)^{3}}}+\sqrt[3]{-\frac{q}{2}-\sqrt{\left(\frac{q}{2}\right)^{2}+\left(\frac{p}{3}\right)^{3}}} .
$$

Essa fórmula, que foi a mais importante descoberta matemática do século XVI, aparentemente apresenta apenas uma raiz. Ocorre que existem três números complexos cujo cubo dá $A=-\frac{q}{2}+$ $\sqrt{\left(\frac{q}{2}\right)^{2}+\left(\frac{p}{3}\right)^{3}}$ (digamos, $\left.A_{1}, A_{2}, A_{3}\right)$ e outros três cujo cubo dá $B=-\frac{q}{2}-\sqrt{\left(\frac{q}{2}\right)^{2}+\left(\frac{p}{3}\right)^{3}}($ digamos, $\left.B_{1}, B_{2}, B_{3}\right)$. Como o produto de qualquer par $A_{i} B_{j}$ que compõe uma solução é tal que $A_{i} B_{j}=$ $\sqrt[3]{A} \sqrt[3]{B}=\sqrt[3]{A B}=\sqrt[3]{P}=-\frac{p}{3}$, deduzimos que a fórmula fornece, realmente, as três raízes da equação $y^{3}+p y+q=0$ pois, escolhido $i$, o $j$ fica automaticamente determinado. Uma vez que a equação em $y$ esteja resolvida, as raízes da equação original em $x$ são obtidas a partir da mudança $x=y-\frac{b}{3 a}$.

Destacamos o radicando comum nas expressões de $A$ e $B$, chamado discriminante e denotado por $\Delta$

$$
\Delta=\left(\frac{q}{2}\right)^{2}+\left(\frac{p}{3}\right)^{3}
$$

que desempenha papel importante na determinação da natureza das raízes da equação, como será visto na sequência.

Como ilustração, vamos aplicar a fórmula à equação ${ }^{10} x^{3}+3 x-10=0$. Posto que $p=3$ e $q=-10$, primeiramente calculamos $\Delta=\left(\frac{-10}{2}\right)^{2}+\left(\frac{3}{3}\right)^{3}=26$. Assim $x=\sqrt[3]{5+\sqrt{26}}+\sqrt[3]{5-\sqrt{26}}$.

Observamos que se $\Delta<0$, a aplicação da fórmula exige conhecimentos de Números Complexos. Retrocedendo à época de sua descoberta, podemos dizer que, realmente, o caso $\Delta<0$ foi o embrião dos posteriores estudos que conduziram ao desenvolvimento dos Números Complexos [7]. Portanto, seria natural que, tanto Cardano quanto Tartaglia não soubessem lidar com essa situação, o que pode ser evidenciado a partir de correspondências trocadas por eles, e que vale a pena aqui reproduzir. Em carta datada de 4 de agosto de 1539, Cardano assim escreveu para Tartaglia: ,

\footnotetext{
${ }^{10}$ Em carta enviada a Tartaglia no dia 9 de abril de 1539, Cardano pediu-lhe que resolvesse explicitamente esta equação, pois o mesmo ainda não havia conseguido decifrar a fórmula que Tartaglia lhe havia passado na forma de versos. Tartaglia respondeu com outra carta em 23 de abril de 1539, resolvendo também, adicionalmente, a equação $x^{3}+x-11=0[19, \mathrm{p} 158]$
} 
Escrevo para dizer-lhe que estou bem e que lhe escrevi muitas outras cartas, às quais, porém, o senhor não se dignou responder [...] ainda mais que lhe pedi a solução de diversos quesitos que ficaram sem resposta, e um deles é o quesito de cubo igual a coisas e número $\left[x^{3}=p x+q\right]$. É verdade que eu entendi a regra, mas quando o cubo da terceira parte das coisas $\left[(p / 3)^{3}\right]$ excede $^{11} 11$ o quadrado da metade do número $\left[(q / 2)^{2}\right]$, então não posso colocar depois deles a equação, como aparece. Portanto, me agradaria muito se o senhor resolvesse essa equação: um cubo igual a nove coisas mais dez $\left[x^{3}=9 x+10\right]$. Realmente, o senhor me faria um favor enorme [19, p172].

Nõ querendo admitir seu desconhecimento sobre a questão, Tartaglia respondeu para Cardano da seguinte forma: ,

Senhor Hierônimo [Gerolamo], recebi a sua carta, na qual me escreve que entendeu o capítulo de cubo igual a coisas e número $\left[x^{3}=p x+q\right]$, mas que o cubo da terceira parte das coisas $\left[(p / 3)^{3}\right]$ excede o quadrado da metade do número $\left[(q / 2)^{2}\right]$, e assim, não pode continuar a equação, e que, por essa razão, me pede que lhe mande resolvido esse capítulo de um cubo igual a nove coisas mais dez $\left[x^{3}=9 x+10\right]$. E, portanto, respondo-lhe e digo que não pegou o caminho correto para resolver esse capítulo; ao contrário, pegou um caminho totalmente errado [19, pp173-174].

Analogamente ao que ocorre com a equação do $2^{\circ}$ grau, dependendo do sinal do discriminante $\Delta$, teremos distintas possibilidades para as raízes. De fato, pode-se mostrar que [10]:

1. Se $\Delta<0$, a equação tem uma raiz real e duas complexas conjugadas;

2. Se $\Delta=0$, a equação tem três raízes reais, sendo uma delas repetida;

3. Se $\Delta=0$, a equação tem três raízes reais e distintas.

A seguir, ilustraremos os três casos anteriores, utilizando figuras construídas no GeoGebra.

Na Figura 2, movendo os controles deslizantes de $p$ e de $q$, de tal forma que o discriminante $\Delta$ mantenha-se positivo, vemos que a função polinomial $f(x)=x^{3}+p x+q$ (Janela de Álgebra) intersecta o eixo $x$ sempre em um único ponto ( $A$, na Janela de Visualização), o que significa que a equação $x^{3}+p x+q=0$ possui apenas uma raiz real.

\footnotetext{
${ }^{11}$ No sentido de se obter $\Delta<0$.
} 


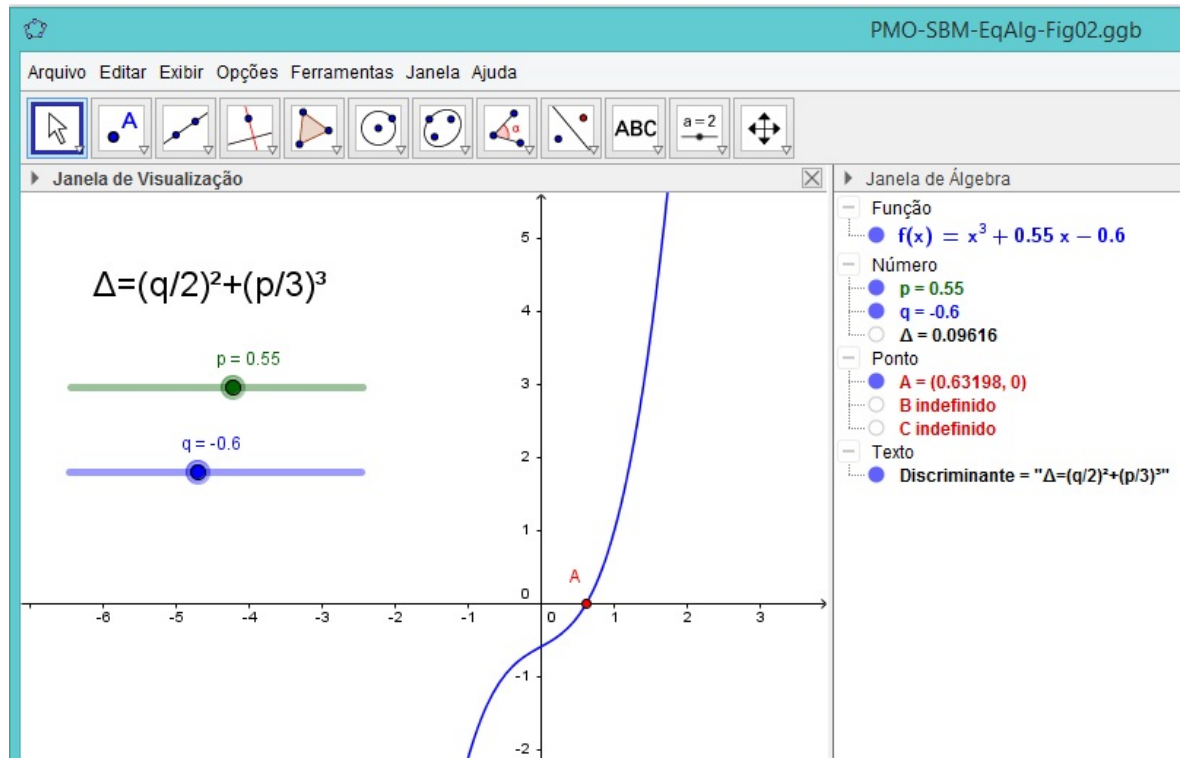

Figura 2: Caso $\Delta>0$

Na Figura 3, movendo os controles deslizantes de $p$ e de $q$, até conseguir que o discriminante $\Delta$ fique nulo, vemos que a função polinomial $f(x)=x^{3}+p x+q$ (Janela de Álgebra) intersecta o eixo $x$ sempre em dois pontos ( $A$ e $B \equiv C$, na Janela de Visualização), sendo que em $A$ existe uma raiz simples e em $B \equiv C$ a raiz é dupla, pois a curva tangencia o eixo $x$.

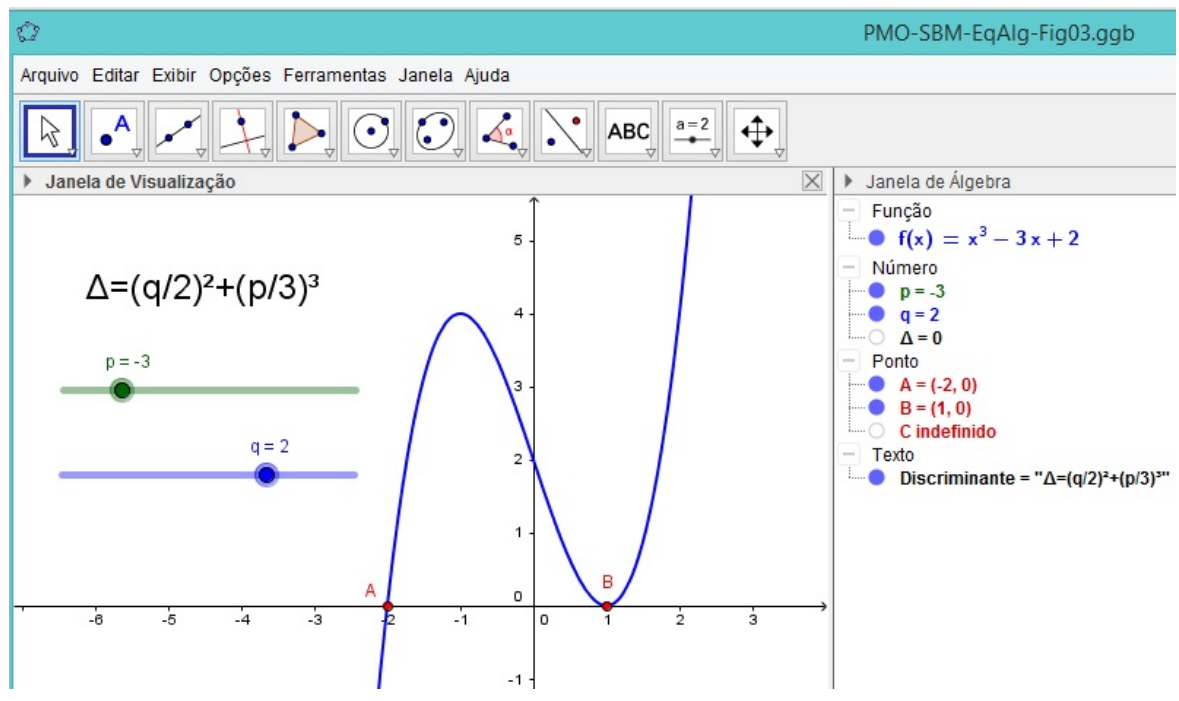

Figura 3: Caso $\Delta=0$ 
Finalmente, na Figura 4, deslizando $p$ e $q$ de tal forma que o discriminante $\Delta$ mantenha-se negativo, vemos que a função polinomial $f(x)=x^{3}+p x+q$ (Janela de Álgebra) intersecta o eixo $x$ sempre em três pontos ( $A, B$ e $C$ na Janela de Visualização), o que significa que a equação $x^{3}+p x+q=0$ possui três raízes reais distintas.

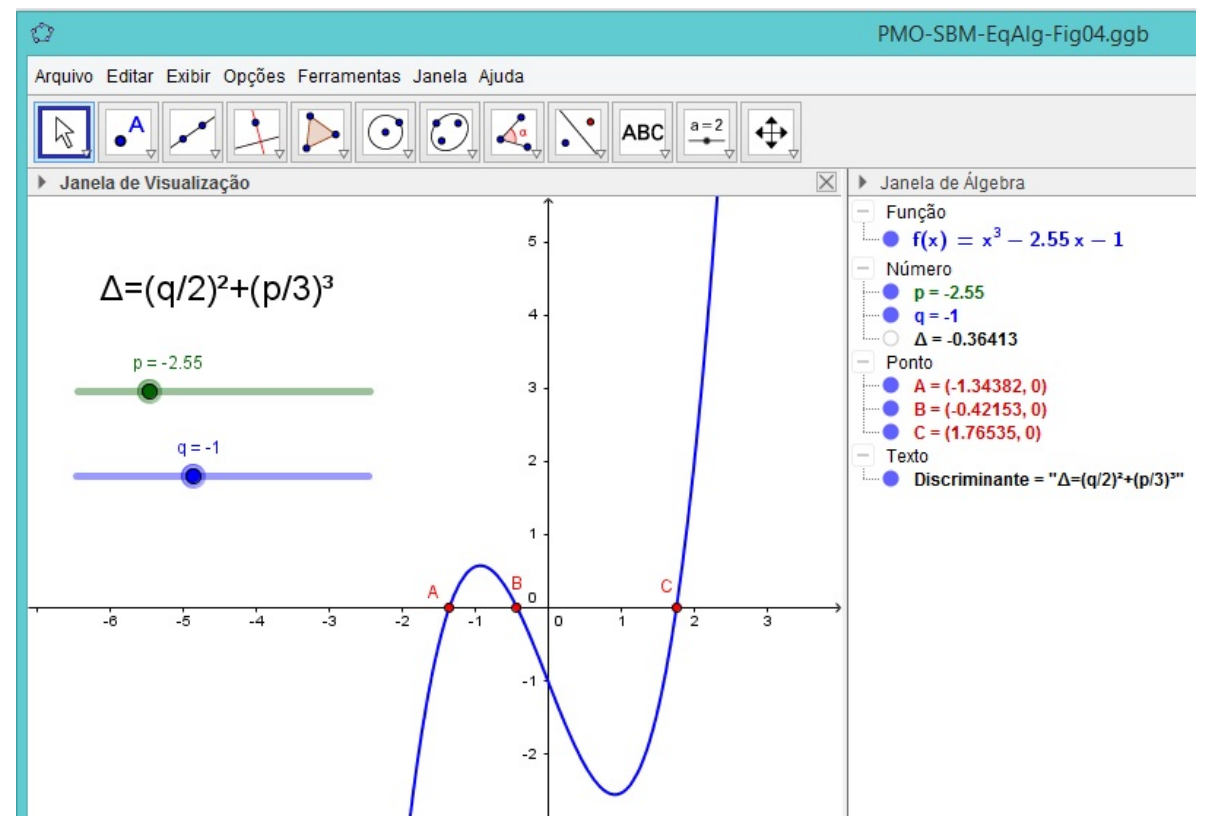

Figura 4: Caso $\Delta<0$

\section{O método da bisseção}

Esta seção apresenta parte da proposta deste trabalho, que é a implementação de um método numérico como estratégia didática no ensino de Equações Algébricas, ideia exposta em [14].

O Método da Bisseção ${ }^{12}[16]$ é estudado em disciplinas de diversos cursos superiores, não fazendo parte dos conteúdos matemáticos abordados no Ensino Básico. Trata-se de um método numérico simples e intuitivo, podendo ser aplicado na busca de zeros de funções contínuas. O método é baseado no Teorema do Valor Intermediário [11], que garante que se $f:[a, b] \rightarrow \mathbb{R}$ é uma função contínua e se $f(a) \cdot f(b)<0$, então $f$ possui pelo menos um zero em $(a, b)$, isto é, existe $\bar{c} \in(a, b)$ tal que $f(\bar{c})=0$.

O algoritmo fornece uma sequência de números $\left(c_{n}\right)$ que converge para a solução procurada $\bar{c}$, da seguinte forma:

1. Se $f(a) \cdot f(b)<0$ então existe ao menos uma raiz da equação $f(x)=0$ no intervalo $(a, b)$.

\footnotetext{
${ }^{12}$ Por se tratar de proposta para o Ensino Médio, deixaremos de lado o Método de Newton, mesmo possuindo mais rapidez de convergência, pelo fato de exigir conhecimentos de "Derivada". Para nossos propósitos didáticos, a simplicidade do método utilizado é mais relevante que a velocidade de convergência.
} 
2. Calcula-se $c=\frac{a+b}{2}$, média aritmética entre $a$ e $b$.

3. Se $f(c)=0$ então $c$ é raiz.

4. Se $f(a) \cdot f(c)<0$ então a raiz estará no intervalo $(a, c)$.

5. Se $f(a) \cdot f(c)>0$ então a raiz estará no intervalo $(c, b)$.

6. Repete-se, iterativamente, o procedimento anterior, até que se chegue à aproximação desejada.

Chamando o intervalo inicial $(a, b)$, da $1^{a}$ etapa, de $\left(a_{1}, b_{1}\right)$, e seu ponto médio $c$ de $c_{1}$, os elementos da segunda etapa de $\left(a_{2}, b_{2}\right)$ e $c_{2}, \ldots$, os elementos da $n-$ sima etapa de $\left(a_{n}, b_{n}\right)$ e $c_{n}$, obtemos três sequências, $\left(a_{n}\right),\left(b_{n}\right)$ e $\left(c_{n}\right)$, tais que

$$
\begin{gathered}
a=a_{1} \leq a_{2} \leq a_{3} \leq \cdots \leq a_{n-1} \leq a_{n}<b \\
b=b_{1} \geq b_{2} \geq b_{3} \geq \cdots \geq b_{n-1} \geq b_{n}>a \\
a_{n} \leq c_{n} \leq b_{n}
\end{gathered}
$$

e

$$
\left|c_{n}-\bar{c}\right|<b_{n}-a_{n}=\frac{b-a}{2^{n-1}}
$$

para todo número natural $n$. Logo, se $n$ for suficientemente grande, teremos que $c_{n}$ estará próximo de $\bar{c}$. De fato, tem-se o seguinte

Teorema 1. Se $f:[a, b] \rightarrow \mathbb{R}$ é uma função contínua e se $f(a) \cdot f(b)<0$, então o Método da Bisseção gera uma sequência $\left(c_{n}\right)$ que converge para $\bar{c} \in(a, b)$, onde $f(\bar{c})=0$.

Em razão de estarmos trabalhando exclusivamente com polinômios, podemos utilizar o Teorema de Bolzano [8], não havendo necessidade de falar aos estudantes do Ensino Médio sobre o Teorema do Valor Intermediário, que é mais geral e aplica-se a qualquer função contínua.

Para exemplificar, mostraremos como se pode calcular a raiz da equação $P(x)=x^{3}+2 x-2=0$ no intervalo $[0,1]$, implementando o Método da Bisseção em uma planilha do GeoGebra, com aproximação de 4 casas decimais. Como $P(0) \cdot P(1)<0$, sabemos que a equação admite uma raiz real no intervalo $(0,1)$. Na utilização da construção representada na Figura 5 , tem-se que:

1. Na Célula $A 1$ o usuário deverá preencher o valor do extremo inferior do intervalo $[a, b]$. Na figura, foi escolhido $a=0$.

2. Na Célula $B 1$ o usuário deverá preencher o valor do extremo superior do intervalo $[a, b]$. Na figura, foi escolhido $b=1$.

3. A Célula $C 1$ foi criada para ser automaticamente preenchida com a média aritmética entre $a$ e $b$, isto é, $C 1=\frac{A 1+B 1}{2}$.

4. As Células $D 1, E 1$ e $F 1$ foram criadas para ser automaticamente preenchidas com os valores $P(a), P(b)$ e $P(c)$, isto é, $D 1=P(A 1), E 1=P(B 1)$ e $F 1=P(C 1)$. 


\begin{tabular}{|c|c|c|c|c|c|c|}
\hline \multicolumn{7}{|l|}{32} \\
\hline \multicolumn{7}{|c|}{ Arquivo Editar Exibir Opções Ferramentas Janela Ajuda } \\
\hline 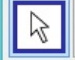 & 田的, $\{1,2\}$ & $\sum_{\nabla}$ & & & & \\
\hline \multicolumn{7}{|l|}{1} \\
\hline & A & B & c & D & $\mathrm{E}$ & $\mathrm{F}$ \\
\hline 1 & 0 & 1 & 0.5 & -2 & 1 & -0.875 \\
\hline 2 & 0.5 & 1 & 0.75 & -0.875 & 1 & -0.0781 \\
\hline 3 & 0.75 & 1 & 0.875 & -0.0781 & 1 & 0.4199 \\
\hline 4 & 0.75 & 0.875 & 0.8125 & -0.0781 & 0.4199 & 0.1614 \\
\hline 5 & 0.75 & 0.8125 & 0.7813 & -0.0781 & 0.1614 & 0.0393 \\
\hline 6 & 0.75 & 0.7813 & 0.7656 & -0.0781 & 0.0393 & -0.02 \\
\hline 7 & 0.7656 & 0.7813 & 0.7734 & -0.02 & 0.0393 & 0.0095 \\
\hline 8 & 0.7656 & 0.7734 & 0.7695 & -0.02 & 0.0095 & -0.0052 \\
\hline 9 & 0.7695 & 0.7734 & 0.7715 & -0.0052 & 0.0095 & 0.0021 \\
\hline 10 & 0.7695 & 0.7715 & 0.7705 & -0.0052 & 0.0021 & -0.0015 \\
\hline 11 & 0.7705 & 0.7715 & 0.771 & -0.0015 & 0.0021 & 0.0003 \\
\hline 12 & 0.7705 & 0.771 & 0.7708 & -0.0015 & 0.0003 & -0.0006 \\
\hline 13 & 0.7708 & 0.771 & 0.7709 & -0.0006 & 0.0003 & -0.0002 \\
\hline 14 & 0.7709 & 0.771 & 0.7709 & -0.0002 & 0.0003 & 0.0001 \\
\hline 15 & 0.7709 & 0.7709 & 0.7709 & -0.0002 & 0.0001 & 0 \\
\hline
\end{tabular}

Figura 5: Cálculo da raiz de $x^{3}+2 x-2=0$ em uma planilha do GeoGebra.

5. As Células $A 2$ e $B 2$ foram criadas para ser automaticamente preenchidas com os extremos do intervalo da segunda iteração ${ }^{13}$.

6. As Células $C 2, D 2, E 2$ e $F 2$ foram criadas para ser automaticamente preenchidas como na Linha 1.

7. A partir da Linha 3, cada Linha $n+1$ deverá ser preenchida como na Linha $n$, devendo o usuário, para este fim, utilizar o recurso de Copiar e Colar.

8. Quando aparecer zero na Coluna $F$, a raiz da equação será mostrada na Coluna $C$ da mesma linha. No presente caso, o zero aparece na Célula $F 15$ e a raiz $\bar{c}=0,7709$ na Célula $C 15$.

\section{Cotas inferior e superior das raízes}

No exemplo anterior, foi dado o intervalo onde se buscava a solução, que é fundamental para aplicar o Método da Bisseção à equação $P(x)=0$. Assim, será importante encontrar números $a$ e $b$, que serão, respectivamente, cotas inferior e superior das raízes reais da equação considerada, isto é, são extremos do intervalo fechado $[a, b]$ que contém o conjunto de todas as raízes reais de $P(x)=0$.

Como estamos buscando raízes da equação $P(x)=a_{n} x^{n}+a_{n-1} x^{n-1}+a_{n-2} x^{n-2}+\cdots+a_{1} x+a_{0}=0$, podemos considerar, sem perda de generalidade, que $a_{n}>0$. Dividindo $P(x)$ por $x-b$, e fazendo uso do Teorema do Resto [8], podemos escrever

$$
P(x)=(x-b)\left(a_{n-1} x^{n-1}+b_{n-2} x^{n-2}+b_{n-3} x^{n-3}+\cdots+b_{1} x+b_{0}\right)+P(b)=0 .
$$

Se todos os números $b_{n-2}, b_{n-3}, \ldots, b_{1}, b_{0}$ e $P(b)$ forem positivos, então $P(x)$ será positivo para todo $x$ maior que $b$, portanto, $b$ será uma cota superior positiva para as raízes de $P(x)$. Fazendo

\footnotetext{
${ }^{13} \mathrm{Na}$ programação dessas células fez-se uso do comando booleano "Se [condição, objeto $X$, objeto $Y$ ]".
} 
uso do algoritmo de Ruffini-Horner [8], dividimos $P(x)$ sucessivamente por $x-1, x-2, \ldots$ (estamos testando números inteiros positivos, a partir de 1), até conseguir que a linha fique toda formada por números positivos, isto é, até encontrar um inteiro positivo $b$ para o qual o quociente da divisão de $P(x)$ por $x-b$ apresente apenas coeficientes positivos.

Por outro lado, observando que as equações $P(x)=0$ e $P(-x)=0$ têm suas raízes diferindo apenas pelo sinal, podemos aplicar o mesmo raciocínio anterior para achar uma cota inferior $(a)$ menor que zero, buscando uma cota superior $(-a)$ para $P(-x)=Q(x)=a_{n}(-x)^{n}+a_{n-1}(-x)^{n-1}+$ $a_{n-2}(-x)^{n-2}+\cdots+a_{1}(-x)+a_{0}=0$. Observamos que, como os cálculos para se obter uma cota superior consideraram o coeficiente do termo de mais alto grau positivo, caso $n$ seja ímpar trabalharemos com $-P(-x)=-Q(x)=0$.

Para exemplificar, criamos uma figura no GeoGebra para encontrar cotas superior e inferior para as raízes da equação cúbica $P(x)=A x^{3}+B x^{2}+C x+D=0$. Na Janela de Visualização da Figura 6, os controles deslizantes $A, B, C$ e $D$ definem os coeficientes da equação, cujos valores são transportados dinamicamente para a Linha 1 da planilha. Ao usuário, basta digitar valores inteiros positivos nas células da Coluna $A$, até que a linha apresente todos os valores positivos, após cálculos realizados pelo algoritmo de Ruffini-Horner. Nesse caso, o último valor da Coluna $A$ será uma cota superior. Para a cota inferior, consideramos $-P(-x)=-Q(x)=A x^{3}-B x^{2}+C x-D=0$ e repetimos o procedimento. Nesse caso, o simétrico do valor que aparece na linha positiva será uma cota inferior. A Figura 6 traz o acima descrito para a equação $2 x^{3}-3 x^{2}-11 x-6=0$. Aqui, temos $P(x)=2 x^{3}-3 x^{2}-11 x-6=0$ e $-P(-x)=-Q(x)=2 x^{3}+3 x^{2}-11 x+6=0$. A cota superior aparece na Célula $A 5$ e o simétrico da cota inferior na Célula $A 9$. As cotas são $a=-2 \mathrm{e}$ $b=4$.

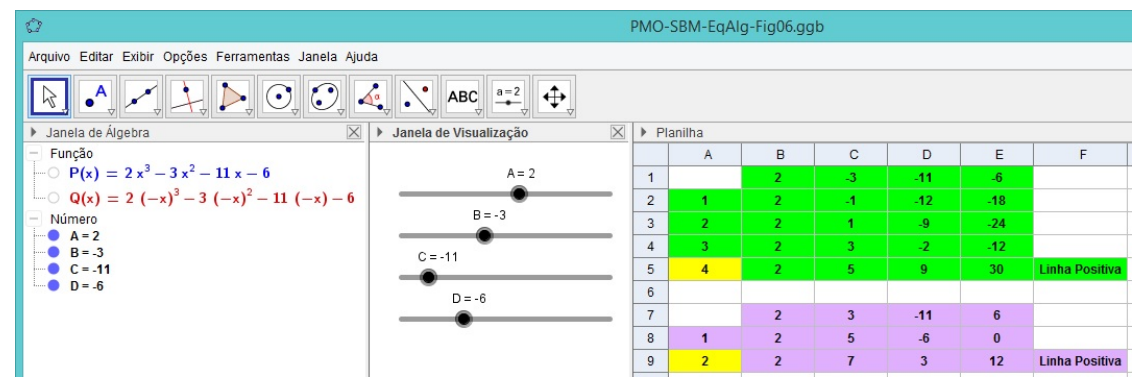

Figura 6: Cotas para as raízes da equação $2 x^{3}-3 x^{2}-11 x-6=0$.

\section{Utilizando mais recursos do GeoGebra}

Após apresentarmos o Método da Bisseção, nenhuma representação gráfica do problema de encontrar zeros de polinômios foi ainda proposta. Essa é, porém, uma abordagem muito esclarecedora, que não podemos deixar de lado. E, para isso, continuaremos utilizando o aplicativo GeoGebra, que possui uma grande variedade de recursos. Lembramos que nosso objetivo não é simplesmente encontrar a resposta, pois, como já vimos, o GeoGebra a produz de forma imediata. O que realmente queremos é mostrar ao estudante como um método numérico permite chegar à solução, que é base do algoritmo que produz a resposta rápida dada pelo aplicativo. Afinal, o GeoGebra tem na sua formulação métodos numéricos! 


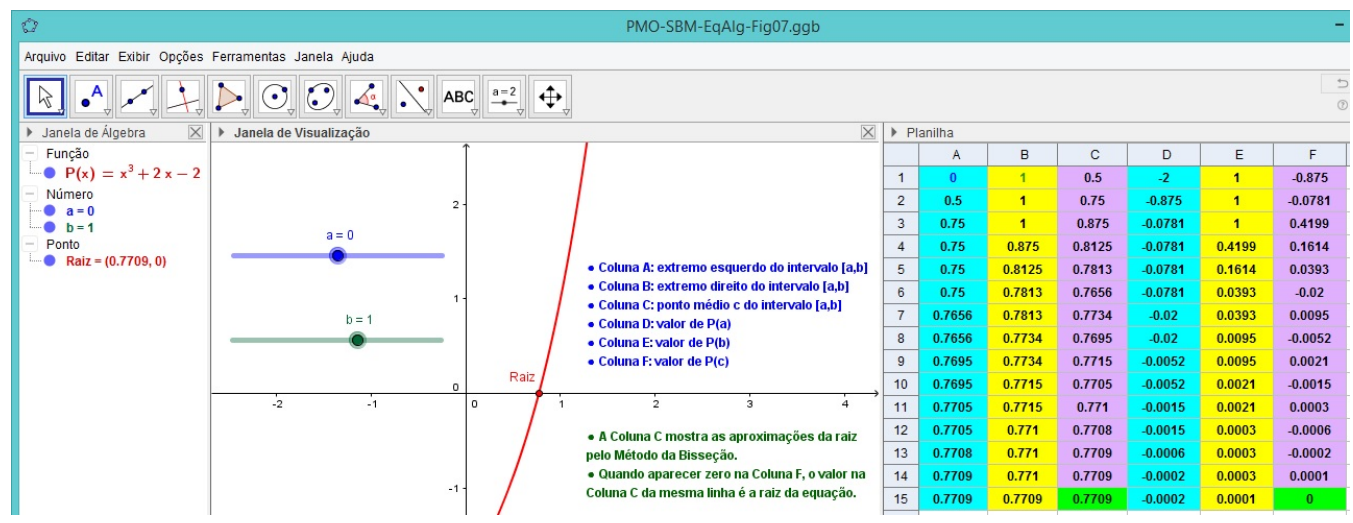

Figura 7: Cálculo da raiz e Visualização de $P(x)=x^{3}+2 x-2=0$ no GeoGebra.

Retornando à equação $P(x)=x^{3}+2 x-2=0$, onde apenas utilizamos uma planilha (Figura 5), agora encontraremos a raiz pelo Método da Bisseção, com aproximação de 4 casas decimais, fazendo uso da representação gráfica, podendo, ainda, variar as cotas inferior $(a)$ e superior $(b)$. Na Figura 7 encontram-se representadas duas janelas e uma planilha. Na Janela de Álgebra, podemos ver a equação a ser resolvida, as cotas e a raiz. Na Janela de Visualização encontra-se a representação gráfica de $P(x)=x^{3}+2 x-2$ e, na Coluna $C$ da Planilha, os valores obtidos pelas iterações do Método da Bisseção.

Movendo os controles deslizantes de $a$ e de $b$ na Janela de Visualização, podemos verificar dinamicamente diferentes sequências que convergem para a solução.

É provável que um estudante mais atento pergunte ao professor o que ocorre quando existe mais de uma raiz real no intervalo considerado, e qual delas o Método da Bisseção encontrará. Uma solução para este problema não é abordada neste texto, mas o professor poderá ilustrar o que acontece em uma situação concreta. Por exemplo, encontrar as raízes reais da equação $2 x^{3}-3 x^{2}-11 x-6=$ 0 . Sabemos que as três raízes reais situam-se no intervalo $[-2,4]$ (ver Figura 6). Utilizando inicialmente $a=-2$ e $b=4$, obtém-se, pelo Método da Bisseção, uma sequência que converge para a solução $c=3,3860$, que aparece na Célula C17 da Figura 8.

Em seguida exploramos variações para o intervalo $[a, b]$. Os controles deslizantes $a$ e $b$ podem ser movimentados livremente, mostrando dinamicamente os resultados interligados nas diversas janelas do GeoGebra, com as correspondentes sequências convergindo para cada uma das raízes. Para $a=-0,9$ e $b=0,1$, a sequência converge para a solução $c=-0,8860$, como mostra a Célula C13 da Figura 9.

Para $a=-2$ e $b=-0,9$, a sequência converge para a solução $c=-1$, como mostra a Célula $C 13$ da Figura 10.

Acreditamos que os diversos resultados encontrados nesta atividade sejam suficientes para mostrar aos estudantes que a convergência para cada raiz dependerá das cotas $a$ e $b$ escolhidas na aplicação do Método da Bisseção.

\section{Considerações finais}




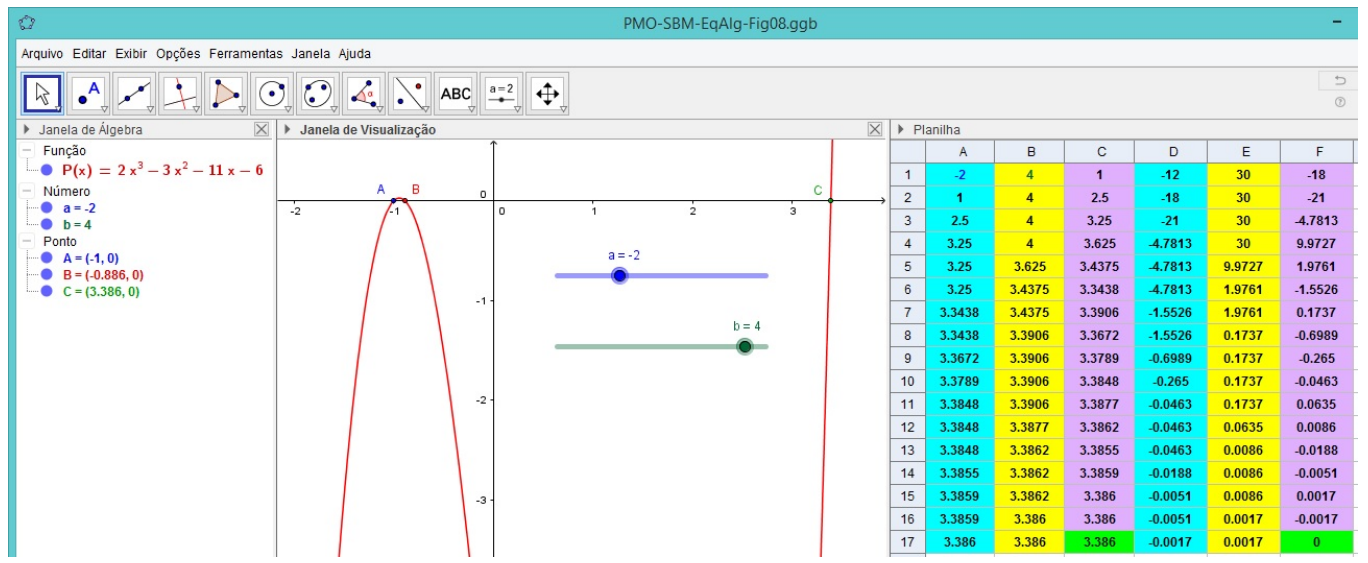

Figura 8: Cálculo da raiz de $2 x^{3}-3 x^{2}-11 x-6=0$ no GeoGebra, utilizando $a=-2$ e $b=4$.

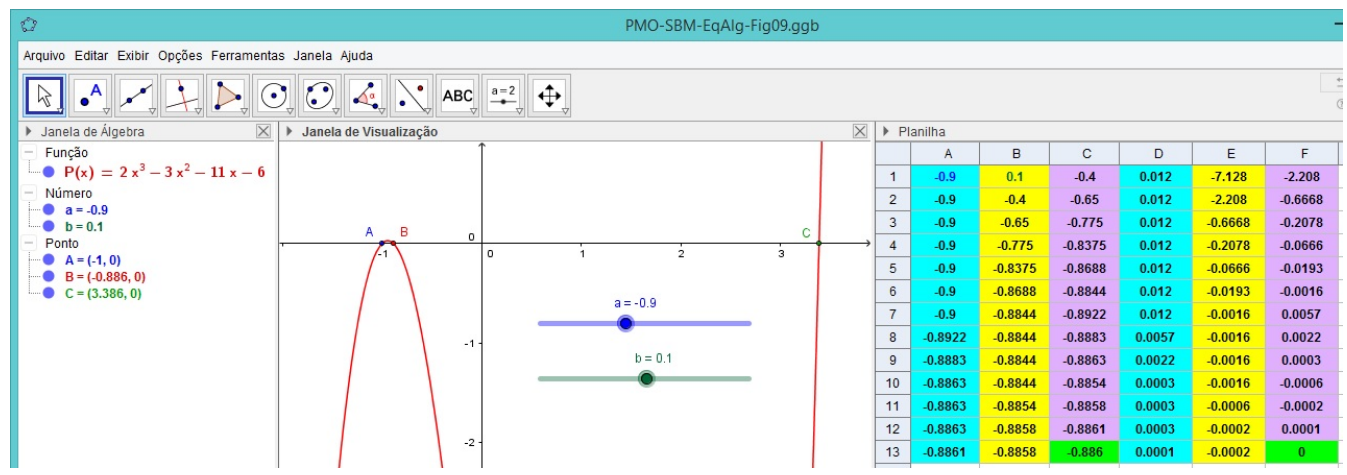

Figura 9: Cálculo da raiz de $2 x^{3}-3 x^{2}-11 x-6=0$ no GeoGebra, utilizando $a=-0,9$ e $b=0,1$.

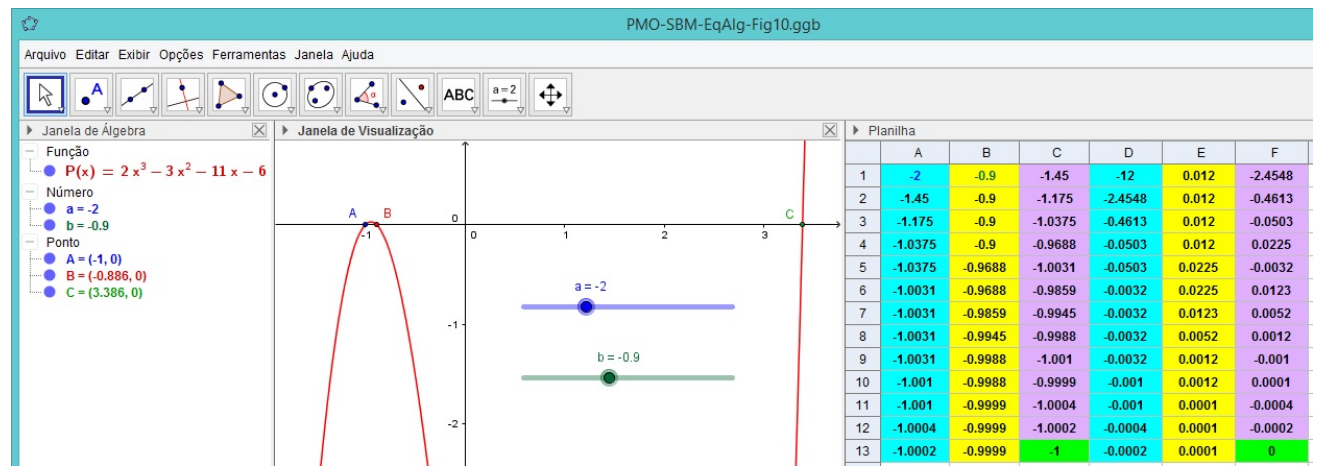

Figura 10: Cálculo da raiz de $2 x^{3}-3 x^{2}-11 x-6=0$ no GeoGebra, utilizando $a=-2$ e $b=-0,9$. 
O Estudo de Equações Algébricas no Ensino Médio, da forma como habitualmente é desenvolvido, é bastante restrito quanto às possibilidades de aplicação, pois praticamente fornece apenas ferramentas para que se encontrem raízes racionais. Nossa proposta de acrescentar o Método da Bisseção ao conteúdo clássico permite ampliar consideravelmente a visão que o estudante pode ter sobre o tema. Além disso, fazendo uso de aplicativos, como o GeoGebra, é possível facilmente implementar o método, de forma que a solução numérica seja encontrada rapidamente. A versatilidade do GeoGebra ao interagir a Janela de Álgebra, a de Visualização e a Planilha, dando diversas visões do mesmo objeto de estudo, ao mesmo tempo que modifica dinamicamente as três opções, é um poderoso e indispensável recurso que deve ser incentivado como auxiliar no processo de ensino e aprendizagem. Além do método numérico e do uso da tecnologia educacional, também acreditamos que a inserção da história nas aulas de matemática pode trazer benefícios nos resultados que se deseja obter. A disputa pela autoria da fórmula que resolve equações algébricas do $3^{\circ}$ grau, bem como sua elegante demonstração, não deveriam ser omitidas dos estudantes.

Para finalizar, apresentamos na Figura 11 a solução feita no GeoGebra do problema que deu início a este trabalho, que foi o de calcular a taxa de juro do financiamento de um carro. Aqui, por esperarmos uma taxa de juro acima de 0, 0099, consideramos a cota inferior como 0, 01 e a superior como 0,06, para ganharmos rapidez na convergência.

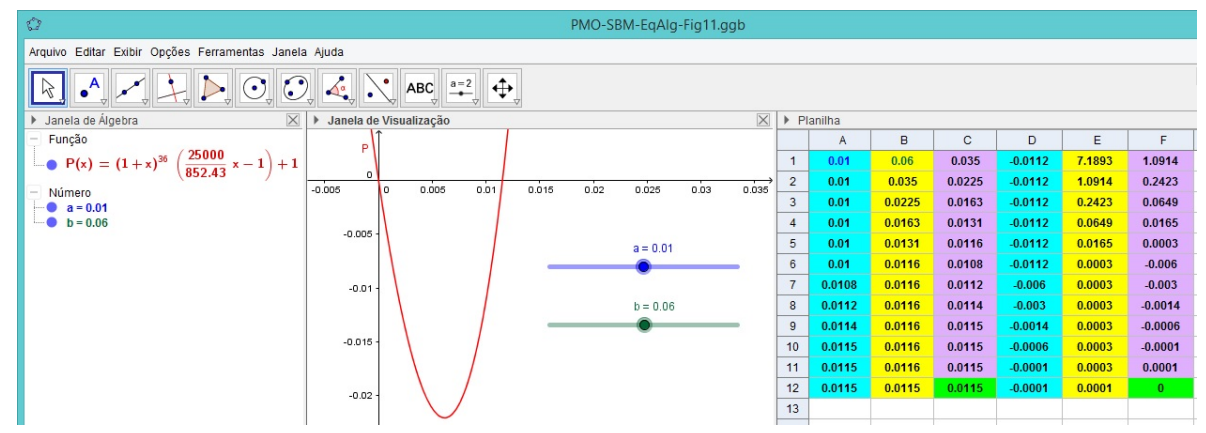

Figura 11: Janelas do Cálculo da Taxa de Juro de um Financiamento.

A Figura 12 ilustra que se pode conseguir, graficamente, uma aproximação de quatro ou mais casas decimais da raiz apresentada na planilha. Isto se obtém utilizando indefinidamente o comando "Ampliar" da Janela de Visualização (último botão à direita da Barra de Ferramentas). Esse recurso permite ver que a raiz está à direita do valor obtido pelas iterações na planilha, e acrescenta outra forma de se decidir sobre o intervalo a ser considerado para consequentes iterações pela Bisseção. Esta abordagem pode ser didaticamente útil para reforçar o caráter da precisão relativa dos métodos numéricos. 


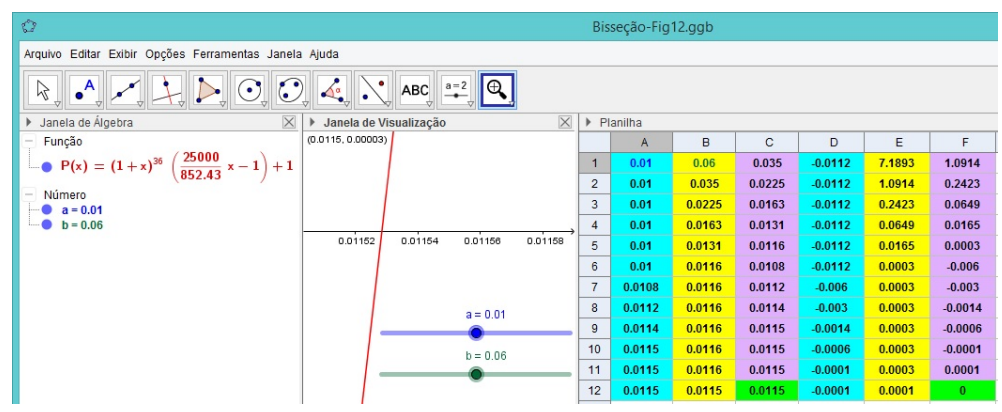

Figura 12: Visualização Gráfica da Aproximação Numérica.

\section{Referências}

[1] Abbeg, T. P. Equações Algébricas no Ensino Médio: História, Resolução Numérica e Tecnologia Educacional. Dissertação, Mestrado Profissional em Rede Nacional - Profmat. Universidade Tecnológica Federal do Paraná, Curitiba, 2014.

[2] Cardano, G. Artis Magnae, sive de regulis algebraicis. Johannem Petreium, Nuremberg, Alemanha, 1545.

[3] Cardano, G. The Rules of Algebra (Ars Magna). Trad. Richard Witmer. Dover, New York, 2007.

[4] Carneiro, J. P. Q. Equações Algébricas de Grau Maior que Dois: Assunto para o Ensino Médio? Revista do Professor de Matemática, n. 40, pp31-40, 1999.

[5] Feldman, R. W. The Cardano-Tartaglia Dispute. The Mathematics Teacher, vol. 54, n. 3, pp160-163, 1961.

[6] Ferrari, L.; Tartaglia, N. Cartelli di Sfida Matematica. Reprodução fac-similar das edições originais de 1547 e 1548. Bréscia, La Nuova Cartográfica, 1974.

[7] Garbi, G. G. O Romance das Equações Algébricas. Makron Books, São Paulo, 1997.

[8] Iezzi, G. Fundamentos de Matemática Elementar, vol. 6: Complexos, Polinômios, Equações. Atual Editora, São Paulo, 2005.

[9] Knudsen, C. A. A Teoria das Equações Algébricas. Revista do Professor de Matemática, n. 7, pp26-31, 1985.

[10] Lima, E. L. A Equação do Terceiro Grau. Revista Matemática Universitária, n. 5, pp9-23, 1987.

[11] Lima, E. L. Análise Real, volume 1. Coleção Matemática Universitária. IMPA, Rio de Janeiro, 2012.

[12] Moreira, C. G. T. A. Uma Solução das Equações do $3^{o}$ e $4^{o}$ Graus. Revista do Professor de Matemática, n. 25, pp23-28, 1994.

[13] Morgado, A. C.; Wagner, E.; Zani, S. C. Progressões e Matemática Financeira. SBM, Rio de Janeiro, 2015.

[14] Ortega Junior, R. R.; Abbeg, T. P. Bisseção no Ensino de Equações. Revista do Professor de Matemática, n. 89, pp47-49, 2016. 
[15] Polcino Milies, C. A Solução de Tartaglia para a Equação do Terceiro Grau. Revista do Professor de Matemática, n. 25, pp15-22, 1994.

[16] Satuf, F. O Método da Bisseção. Revista Matemática Universitária, n. 36, pp39-49, 2004.

[17] Tartaglia, N. Quesiti et Inventioni Diverse. Veneza, 1546.

[18] Tartaglia, N. Questions et Inventions Diverses. Livre IX ou l'invention de la résolution des équations du troisième degré. Hermann, Paris, 2010.

[19] Toscano, F. A Fórmula Secreta. Editora da Unicamp, Campinas, 2012.

Rubens Ortega

Universidade Tecnológica Federal do Paraná - Curitiba-PR

<rubensortega@utfpr.edu.br>

Thiago Abbeg

Centro Universitário Claretiano - Curitiba-PR

<thiagoabbeg@yahoo.com.br>

Recebido: 2016

Publicado: 2016 